the adverse conditions prevailing in the homoiotransplanted tissue. But in the latter case a weakening in the effect of the growth substance takes place. The injurious action of body fluids in the other individual detracts from the efficacy of the growth substance.

9. On the basis of these observations, we may formulate the following conception of the relations between different tissues: A certain metabolic activity on the part of parenchyma of various organs determines the attitude of lymphocytes and of fibroblasts toward the parenchyma. If the activity is normal, lymphocytes do not, or only to a slight extent, enter the parenchyma. The connective tissue is held in a definite state of activity. If within certain limits (which still permit the continued life and even growth of the tissue) the metabolic condition of the parenchyma becomes abnormal, the connective tissue in contact with the parenchyma loses its normal state of activity, produces fibrous bands (and perhaps the fibroblasts invade the parenchyma in larger number) and thus compression is exerted on the parenchyma, and cirrhosis caused and ultimately the transplanted tissue is destroyed. This is one mode in the production of cirrhosis. Under other conditions, the presence of actual defects in the parenchyma may under the guidance of stereotropic stimuli ${ }^{12}$ also produce an invasion by connective tissue and thus lead to cirrhosis. We notice in certain mucous membranes that directly beneath the epithelium the connective tissue is richer in cells than elsewhere. This may, in conformity with the theory just presented, be due to the stimulating effect of the metabolism of the epithelium similar to that exerted by normal parenchyma on the connective tissue after autotransplantation.

A sinilar abnormal metabolic condition of parenchyma may perhaps also be produced in certain states of immunity, and thus it may be explained that in animals immunized against tumor growth, various mononuclear leukocytes invade or surround the tumors. On the basis of our theory, this would be a process secondary to metabolic changes resulting from the effect of substances produced in the animal through immunization and acting on the tumor cells, or directly produced through immunization in these tumor cells, the combination of this inmune substance and the tumor cells influencing certain host cells in a manner comparable to that caused by parenchyma after homoiotransplantation.

Connective tissue and lymphocytes may therefore be regarded as organs of attack, lying quiescent under ordinary conditions, but exerting their efforts as soon as within given limits certain pathologic changes take place. While according to the view commonly held and mainly inaugurated by Weigert (so-called inflammatory) connective tissue growth accompanying round cell infiltration is of a regenerative character, destined to fill out actual defects or to take the place of cells markedly injured and unable to continue a vigorous life, our observation points to the conclusion that also tissues not essentially injured in their vitality and not markedly changed in their morphologic character may as a result of altered metabolic conditions influence lymphocytes and connective tissue in such a manner that cirrhosis and a partial or total destruction of the parenchyma results.

12. Concerning the significance of stereotropic stimuli for the growth of tissues, compare the following paper: Loeb, Leo: Ueber die Regencration des Epithels, Arch. f. Entwicklungsmechanik, 1898, vi, 237, Chapter 13 .

\section{REPORT OF TYPHOID EPIDEMIC OCCUR- RING THREE MONTHS AFTER THE USE OF PROPHYLACTIC VACCINE}

\author{
E. H. TROWBRIDGE, M.D., B. A. FINKLE, M.D. \\ AND
} ELIZABETH M. BARNARD, M.D.

First, Second and Third Assistant Physicians, Respectively, Minnesota School for Feeb?e Minded and Colony for Epileptics

FARIBAULT, MINN.

During the early months of the spring of $1914 \mathrm{sev}-$ eral cases of typhoid fever occurred in the Minnesota School for Feeble Minded and Colony for Epileptics at Faribault. An exhaustive search through the use of the Widal test in some 1,601 inmates disclosed the presence of a positive reaction in twenty-eight. Further investigation by means of isolation tests revealed the fact that eight of these inmates were active carriers.

To prevent the development of new cases, the administration of typhoid prophylactic was begun immediately. A total of 1,520 patients were inoculated in the usual manner. All patients having given positive Widals were isolated until the completion of the prophylactic treatment. New cases ceased to develop following the administration of the prophylactic until the outbreak of the current epidemic.

In order to determine the agglutinating property of the blood, following the prophylactic treatment, specimens of blood were taken from all who were inoculated. Complete results showing reactions in males and females, and the percentage are given in detail in Table 1.

TABLE 1.-RESULTS OF WIDAL TESTS MADE FOLLOWING IMMUNIZATION

\begin{tabular}{l|c|c|c|c|c|c}
\hline & \multicolumn{2}{|c|}{ Male } & \multicolumn{2}{c|}{ Female } & \multicolumn{2}{c}{ Total } \\
\cline { 2 - 4 } & No. & Per Cent. & No. & Per Cent. & No. & Per Cent. \\
\hline Positive reaction.... & 262 & $18.8+$ & 215 & $15.4+$ & 477 & $34.3+$ \\
Atypical reaction.... & 130 & $9.3+$ & 135 & $9.7+$ & 265 & $19.0+$ \\
Negative reaction... & 317 & $22.8+$ & 331 & $23.8+$ & 648 & $46.6+$ \\
\hline Total widals taken & 709 & $51+$ & 681 & $48+$ & 1390 & 100 \\
\hline
\end{tabular}

About the middle of September, three months subsequent to the completion of the administration of the antityphoid vaccine, several patients were admitted to the hospital with symptoms somewhat resembling those of typhoid fever. As these patients had been previously inoculated, a diagnosis of typhoid fever was not justifiable without a more thorough investigation.

On request the State Board of Health kindly consented to assist us in this task. Blood cultures were taken from twelve of the patients with the result that the Bacillus typhosus was found in one instance. This same patient gave a positive Widal reaction following the use of the prophylactic, and also after the onset of the disease. At necropsy typhoid ulceration was found in the ileum, thus verifying the nature of the infection.

Specimens of feces and urine were collected and examined; the Bacillus typhosus was isolated in two cases and the bacillus paratyphosis in three. Widals were made at intervals during the course of the epidemic. Of the forty-six patients examined, thirtynine gave positive Widals, one gave an atypical reaction, two gave agglutinations with the bacillus para- 
typhosis, and three remained negative, although manifesting clinical signs of typhoid fever. Due to oversight, no Widal was made on illustrative Case 3 .

Owing to the complexity of symptoms, including pharyngitis, cultures from the nose and throat were made, which showed the presence of diphtheria bacilli in eleven of the patients. These being of a non-virulent type, prove conclusively that they bore no important relation to the course of the disease.

The typhoid occurring in separate buildings scattered over a wide area made it possible to dismiss the previous carriers as a source of infection, and to focus attention on the food, milk and water supply.

The distribution of food is from a central kitchen, and the milk supply is from the institution dairy. The major portion of the water is obtained from the city water supply, and the remainder from our own artesian well. The general water supply as a source of infection was ruled out, since sewage contamination was found to be improbable.

A spring on the east side of the grounds was considered, since it is possible for it to receive surface drainage, and several of the patients had had access to this water. As the majority of the patients had not partaken of this water, it was evidently not the primary source of infection. No carriers were found among those concerned with the preparation and distribution of food.

Investigation of the conditions existing at the dairy disclosed the fact that the dairyman had an ambulatory case of typhoid. The dairyman's wife was ill at the time; her symptoms were of an indefinite nature and a subsequent Widal was found to be positive. Neither the dairyman nor his wife had previously received prophylactic treatment. A milker, one of the inmates, had been removed to the hospital a few days previous to the investigation of the dairy, and after a prolonged prodromal stage developed clinical typhoid fever. He had returned recently from a short vacation in an adjacent town, where he no doubt contracted the disease. The daily association of the dairyman and his wife with this milker indicates that he was the original source of infection. Precautionary measures were immediately instituted. The dairyman and his wife were relieved from duty, and all the milk was sterilized during the next four weeks. Milk formed part of the diet of every individual who contracted the disease.

After the incubation period of twenty-one days had passed, new cases ceased to develop, showing conclusively that the milk supply was the source of the epidemic.

A total of fifty-seven cases in this epidemic were diagnosed as clinical typhoid. Of this number fortysix were inmates and the remainder were employees. Only one of the employees had received the prophylactic, and three of the inmates were uninoculaterl.

The following typical case histories are presented to illustrate the symptoms as they appeared in the clinical picture.

\section{REPORT OF CASES}

CASE 1.-(No. 7) G. M., girl, aged 18; admitted to the hospital Sept. 23, 1914; discharged Dec. 23, 1914.

Personal History.-Widal test made May 20, 1914, negative; typhoid prophylactic administered May 27, June 6 and 16, 1914. Widal test made July 9, 1914, negative.

Present Illness.-Began with severe headache, anorexia, nausea and vomiting and diarrhea, followed by marked prostration, restlessness and delirium. Tongue coated, sordes marked, abdomen very tender, spleen palpable. Rose spots appeared on the abdomen. Temperature on admission, $101 \mathrm{~F}$, and 104 to 105 in the afternoon for ten days, gradually declining to normal on the thirty-sixth day. Stools contained occult blood on the eighth day, and two fairly large intestinal hemorrhages occurred on the thirteenth day.

On the fourteenth day 3,000 units of diphtheria antitoxin were administered.

Laboratory Findings.-Widal test made Sept. 29, 1914. atypical. Widals made Oct. 3, 1914, and Nov. 1, 1914, positive. Blood culture taken Sept. 30, 1914, negative. Throat culture taken Oct. 10, 1914, showed diphtheria bacilli of a non-virulent type.

CASE 2.-(No. 9) A. E., man, aged 23. Admitted to hospital Sept. 24, 1914. Discharged Nov. 25, 1914.

Personal History.-Widal made May 6, 1914, negative. Typhoid prophylactic administered May 27, June 8 and 22, 1914. Widal made July 1, 1914, positive.

Present Illness.-Complained of headache, sore throat and abdominal distress for about a week before admission to hospital. On admittance had headache, pain in limbs, coated tongue, some abdominal tenderness and anorexia. Constipated during entire course of the disease. Temperature $103.4 \mathrm{~F}$. when received at hospital, and fluctuated between 102 and 104 for eight days. Began to decline the ninth day, reaching normal on the twenty-first day. From twenty-first to thirty-first day the temperature was very irregular, varying from 96.4 to 101.8. It remained subnormal or normal on the forty-seventh and forty-eighth days, and rose to 101 on the forty-ninth day, dropping to normal on the following day. On the seventeenth day of the disease 3,000 units of diphtheria antitoxin were given.

Laboratory Findings.-Widals made Sept. 28, 1914, Nov. 1, 7, 9, 13 and 17, 1914, negative. Widal made Nov. 22, 1914, positive. Blood culture taken Sept. 30, 1914, negative. Throat culture taken Oct. 10, 1914, showed diphtheria bacilli of a non-virulent type.

CAse 3.-(No. 10) M. L., man, aged 24, admitted to hospital Sept. 24, 1914. Died Oct. 16. 1914.

Pcrsonal History.-Widal made May 30, 1914, negative. Typhoid prophylactic administered June 5, 15 and 25, 1914. Widal made Aug. 17, 1914, negative.

Present Illuess.-For a few days before entering hospital the patient was annoyed by frequent epistaxis, diarrhea was marked and pains in the back were severe. Temperature $101.6 \mathrm{~F}$. on admission to hospital, and rose to 104.6 the following day. Temperature remained high until the fifteenth day in the hospital, when there was a sudden drop of 99 , with evidence of perforation. The next day his temperature rose to 105 , the abdomen became tense and the patient died.

Laboratory Findings.-Blood culture taken Sept. 30, 1914, negative. Necropsy showed two small perforations in the ileum and numerous ulcers, some beginning, some mature and others healing.

CASE 4.-(No. 13) C. S., man, aged 21. Admitted to hospital Sept. 26, 1914. Discharged Nov. 25, 1914.

Personal History.-Widal made May 30, 1914, negative. Typhoid prophylactic administered June 5, 15 and 25, 1914 Widal made July 14,1914 , negative. Widal made July 18 , 1914, positive.

Present Illness.--Five days prior to entering hospital there was anorexia, fatigue and pains in the back and neck. Entered hospital September 26 with tongue heavily coated, stools watery and very offensive. Temperature 101 to 103.6 for thirty-three days, when it began to recede and the patient made an uneventful recovery.

Laboratory Findings.-Blood count normal. Blood culture taken Sept. 30, 1914, negative. Widals made Oct. 26 and Nov. 1, 1914, positive. Throat culture taken Oct. 10, 1914, showed diphtheria bacilli of a non-virulent type.

Case 5.-(No. 20) H. T., girl, aged 5, admitted to hospital Sept. 29, 1914. Died Nov. 27, 1914.

Personal History.-Typhoid prophylactic not given.

Present Illness.-Admitted to hospital with bronchitis. Temperature 102 and 98 to 99 for twenty-four days, then rose 
suddenly to 103 with extreme prostration, restlessness and delirium. Sordes marked, abdomen tympanitic, spleen palpable. Temperature ranged from 103 to 105 in the afternoon, until death occurred on the fifty-eighth day.

Laboratory Findings.-Widals made Oct. 12, 1914, and Nov. 1, 1914, negative. Widals made Nov. 7, and Nov. 9, 1914, atypical. Widal made Nov. 13, 1914, positive. Necropsy: Ulcers found in large number in the ilemm.

CASE 6.-(No. 31) C. C., woman, aged 36, admitted to hospital Oct. 5, 1914. Discharged Nov. 25, 1914.

Personal History.-Widal made May 21, 1914, negative. Typhoid prophylactic administered June 2, 12 and 22, 1914. Widal made July 11, 1914, negative.

Present Illness.-Patient suffered from anorexia and general malaise for ten days. Then had a sudden, severe rigor with throbbing headache, severe backache and pain in the chest. Was constipated and had a racking cough. There was a marked tenderness in the right iliac region, tongue coated, sordes marked, rose spots on abdomen. Crepitant râles conld be heard in the bases of both lungs posteriorly, but no dulness was found. Temperature on admission 100.6 and ranged from 103 to 104 in the afternoon for nine days, then gradually declined and reached normal on the sixteenth day.

Laboratory Findings.-Widal made Oct. 26, 1914. atypical. Widals made October 29 and November $1,7,15,17$ and 22, negative. On November 17 agglutination was obtained with the Bacillus paratyphosus, and this bacillus was isolated from the stool.

CASE 7.- (No. 43) C. M., man, aged 17, admitted to loospital Oct. 22, 1914. Discharged Dec. 4, 1914.

Personal History.-Widal made May 6, 1914, negative. Typhoid prophylactic administered April 30, May 11 and 21, 1914. Widal made Aug. 13, 1914, negative.

Present Illness.-Complained of headache and abdominal pain two days previous to admission. On admission the patient was very nervous and covered with profuse perspiration; tongue heavily coated, anorexia, slight abdominal tenderness over splenic area. Spleen became palpable and a few rose spots appeared. Constipated during course of disease.

Laboratory Findings.-Widals made Nov. 1 and Nov. 24, 1914, positive.

The foregoing histories illustrate a more typical course of typhoid than is presented in the majority of the cases. Symptoms common to all cases were anorexia, indefinite pain in back, neck or abdomen, headache and constipation. Acute toxemia and marked prostration were present in but three of the previously inoculated patients. In fact it is worthy of note that many of the classical symptoms of typhoid, such as enlargement of the spleen, rose spots, epistaxis and abdominal tenderness were absent in most cases. However, the course of the disease did not appear to be appreciably shortened. The mortality among the vaccinated was 9.1 per cent., or less than one-half of that occurring among the unvaccinated, which was 23 per cent.

The cause of an epidemic of this nature so shortly after the completion of vaccination with the antityphoid vaccine is a subject for further discussion.

Various reports submitted by recent investigators who have had vast experience in the use of the prophylactic among large numbers of individuals, show that it is not an uncommon occurrence for typhoid fever to develop among a certain proportion of protected persons.

The use of the vaccine by the Germans in 1904 in the Herero campaign in Southern West Africa, resulted in a diminution of the occurrence of cases among the protected to about one-half as compared with that among the unprotected. The mortality among the unprotected was about four timcs as great as among the protected.

Figures computed by Col. R. H. Firth, covering the first six months of the year 1911, relative to vaccinated soldiers in India, show that the case incidence for typhoid was approximately five times greater among the non-protected than among the protected.

In a recent article by Frederick $F$. Russel, ${ }^{1}$ U. S. A., are cited the remarliable results obtained by the use of the prophylactic in the United States army during the past six years. He says:

Table 1 shows that the number of cases in the United States proper has fallen from 3.53 per thousand men to 0.03 in the six years; the death rate has fallen from 0.28 in 1909 to zero in 1913. In both instances the greatest improvement has been during the past three years since the vaccination was made compulsory.

No doubt these extraordinary results were in part due to the fact that camp sanitation was greatly improved and the chance of infection reduced to a minimum.

In the epidemic under consideration about one-half as many cases occurred in the prctected as in the nonprotected, and the mortality among the protected was less than one-half that among the non-protected.

No doubt the proportion of cases among the protected individuals would have been greatly reduced were it not for the fact that the milk, which was the source of infection, was saturated from day to day with typhoid bacilli.

So far as we are able to ascertain from the literature investigated, there is no record of a protected community having had one of the chief sources of food supply contaminated with typhoid bacilli over an extensive period of time, as occurred in this recent epidemic.

Other factors to be considered which might influence the proportion of disease incidence in vaccinated individuals, are as follows:

The possibility of the vaccine used having been overheated during its preparation, which accounted for the unsatisfactory results obtained by those who first experimented with its use. The use of vaccine which has deteriorated from not being kept at the proper temperature, or on which the time limit has expired. Faulty technic in the method of administration. Failure to adhere to the proper time interval between inoculations and to the correct number of bacteria injected.

The vaccine used in inoculating individuals connected with this institution was prepared and furnished entirely by the State Board of Health, used within the specified time limit, and kept at the proper temperature. The method of administration, the dosage, and the time interval were adhered to as recommended by the State Board of Health, except that 10 minims instead of $7 \mathrm{I} / 2$ were given at the first inoculation. Statistics show that 65 to 70 per cent. of exposed individuals have a natural immunity and therefore do not contract the disease.

It is evident from a study of this epidemic, and from the experience of others, that the typhoid vaccine in some cases fails to confer immunity, or confers only partial immunity.

Laboratory investigations by means of the Widal reaction have shown in those cases previously inocu-

1. Russel, F. F.: Antityphoid Vaccination in the Army During 1913, THE Journal A. Mi. A., May 2, 1914, p. 1371 . 
lated with the vaccine, that the blood in most cases has acquired the power of agglutinating typhoid bacilli.

We know that following a clinical attack of typhoid fever the agglutinating property of the blood is present, and that frequently this is lost after varying lengths of time, while the individual possesses as a rule a lifelong immunity. At present the only laboratory index by which immunity is suggested is the appearance of agglutinins in the blood. The occurrence of clinical typhoid fever in those having been previously inoculated proves that the appearance of agglutinins in the blood is not a positive index of immunity.

Of those who developed typhoid fever in this epidemic, 14.3 per cent. gave positive Widals after inoculation, 21.4 per cent. gave atypical reactions, and 64.3 per cent. gave negative reactions.

Negative blood reactions following the use of the prophylactic may be due to one or more of the following factors: incorrect interpretation of the reaction; improper dilution of the serum; the use of freshly isolated cultures, which often resist agglutination; and, dccomposition of the blood, due to incomplete drying of the specimens collected. It is also worthy of note that the agglutinating power of the blood does not remain constant, which is clearly shown in Table 2.

TABLE 2.-RESUI.TS OF WIDALS MADE IN 127 INDIVIDUALS (MALES) AT TEN-DAY INTERVALS FOLLOWING FIRST, SECOND AND THIRD INOCULATIONS

\begin{tabular}{|c|c|c|c|c|c|c|}
\hline & \multicolumn{2}{|c|}{$\begin{array}{l}\text { Reaction Fol- } \\
\text { lowing First } \\
\text { Inocllation }\end{array}$} & \multicolumn{2}{|c|}{$\begin{array}{l}\text { Reaction Fol. } \\
\text { lowing Second } \\
\text { Inoculation }\end{array}$} & \multicolumn{2}{|c|}{$\begin{array}{l}\text { Reaction Fol- } \\
\text { lowing 'Jhirt } \\
\text { Inoculation }\end{array}$} \\
\hline & No. & Per Cent. & No. & Per Cent. & No. & Per Cent. \\
\hline $\begin{array}{l}\text { Positive reaction.... } \\
\text { Atypieal reactioll... } \\
\text { Negalive reaction... }\end{array}$ & $\begin{array}{r}12 \\
9 \\
106\end{array}$ & $\begin{aligned} 9.4+ \\
7.0+ \\
83.5+\end{aligned}$ & $\begin{array}{l}67 \\
15 \\
45\end{array}$ & $\begin{array}{l}52.7+ \\
11.8+ \\
35.4+\end{array}$ & $\begin{array}{l}37 \\
29 \\
68\end{array}$ & $\begin{array}{l}29.1+ \\
17.3+ \\
53.5+\end{array}$ \\
\hline Total Widals taken. & 127 & 100 & 127 & 100 & 127 & 100 \\
\hline
\end{tabular}

Reviewing the findings in the preceding table, it is noted that the positive and atypical reactions begin to appear following the first inoculation. Following the second inoculation, there is a marked increase in the positive and a slight increase in the atypical reactions. The positives following the third inoculation decrease almost 50 per cent., while the atypicals show a steady increase.

\section{CONCLUSIONS}

1. Typhoid fever may be contracted by individuals who have received the prophylactic and who subsequently show a positive Widal.

2. The course of the disease is not appreciably shortencd in vaccinated individuals.

3. The mortality is markedly reduced among protected persons.

4. Protected persons having typhoid fever fail to give many of the classical symptoms of the disease.

5. The development of paratyphoid is not prevented by the use of the typhoid prophylactic.

6 . The degree of immunity conferred by the prophylactic in some cases fails to prevent the development of typhoid when the individual has been subject to repeated exposure.

7. The Widal reaction as a criterion of the presence of immunity is of uncertain value.

Typhoid vaccination applied as a routine measure in all of our hospitals and institutions is the one and only method to pursue to eradicate this ever present, serious, and often fatal disease. As a step in this direction State Boards of Health throughout the country are giving their cooperation and furnishing the vaccine free, to stimulate its use.

To the Minnesota State Board of Health we owe a debt of gratitude for their ever willing and hearty cooperation; their assistance in locating the source of the epidemic; and their promptness in performing the various laboratory tests.

\section{MIEDICAL INSPECTION OF OPEN AIR SCHOOLS}

JOHN AIKMAN, M.D.

Attending Physician, Open Air School

ROCHESTER, N. Y.

Since the introduction, in 1908, of open air schools into the educational system of America, the number of these schools has increased at a surprising rate. To-day every city has an open air school, and the general plan is well known to all.

It is not the object of this paper to deal with the whole subject of this type of school, but with a feature which, up to this time, has received little comment.

As these institutions differ in many ways from the ordinary school, so also does the medical inspection present different problems.

The Edward Mott Moore Open Air School of this city was among the first to be opened in America, and we have been able in the past six years to try out various plans for medical inspection. After many experiments we have developed a system that is meeting requirements. As many physicians have been and will be called on to supervise work of this nature, we have thought that the results of our experiences might be of value to them in undertaking such work.

I will endeavor to outline briefly the work as it is carried on at the present time.

The medical inspection of our school is not under the direction of the city school inspectors, but is provided by the Rochester Public Health Association, which maintains a general dispensary as well. In many ways this removal from the regular school system is an advantage. The child is really taken as a patient of the association, and all problems of physical welfare are settled by physicians from the staff. Thus it is possible to make complete physical examinations, while in the regular schools they are very limited, as the child must be examined dressed. The difficulty of having directions followed in regard to corrections of physical aiiments, which presents such a problem in the regular schools, is here eliminated, as we have, and exercise, the right to exclude or dismiss any children not following out our instructions. Of course such children are allowed to return to their former schools. Under these instructions would come removal of tonsils and adenoids, fitting of glasses, dental work, etc.

The routine now is as follows: All applications for admission are made at the association building, and the examinations are made on a certain afternoon of each week by the medical director of the association. It the case is suitable, recommendations are made for filling the teeth, removal of tonsils and adenoids, treatment of skin conditions and ccrrection of anything that would prevent the child getting the most good out of the school. 